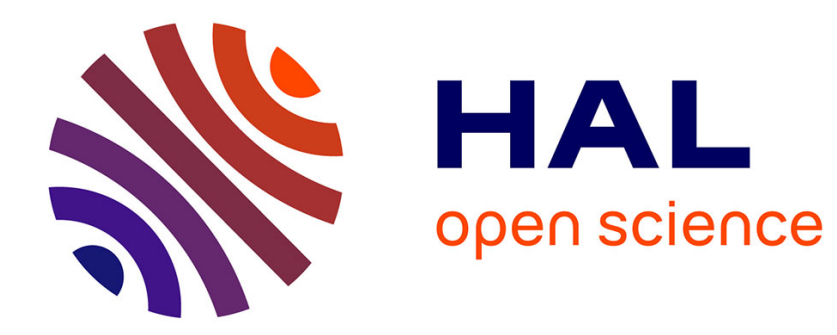

\title{
Effets de l'orthographe dans la prononciation du français L2
}

Fabian Santiago

\section{To cite this version:}

Fabian Santiago. Effets de l'orthographe dans la prononciation du français L2. Journées d'Etudes sur la Parole (JEP) 2018, Jun 2018, Aix-en-Provence, France. pp.160-168. hal-01768620

\section{HAL Id: hal-01768620 \\ https://hal.science/hal-01768620}

Submitted on 29 May 2018

HAL is a multi-disciplinary open access archive for the deposit and dissemination of scientific research documents, whether they are published or not. The documents may come from teaching and research institutions in France or abroad, or from public or private research centers.
L'archive ouverte pluridisciplinaire HAL, est destinée au dépôt et à la diffusion de documents scientifiques de niveau recherche, publiés ou non, émanant des établissements d'enseignement et de recherche français ou étrangers, des laboratoires publics ou privés. 


\title{
Effets de l'orthographe dans la prononciation du français L2
}

\author{
Fabián Santiago ${ }^{1,2,3}$
}

(1) UMR 7023 CNRS, SFL, Université Paris VIII, 75017, Paris, France (2) UMR 7018 CNRS, LPP, Université Sorbonne-Nouvelle, 75005 Paris, France (3) UMR 7110 CNRS, Université Paris Diderot, 75013, Paris, France fabian.santiago-vargasduniv-paris8.fr

\section{RÉSUMÉ}

Nous étudions les effets de deux types de tâches (imitation $v s$ lecture oralisée), le rôle de l'orthographe et le niveau de langue dans la prononciation du français L2 chez 27 étudiants hispanophones. Pour évaluer la prononciation en L2, nous analysons les distances de Levenshtein entre les transcriptions phonétiques de la forme canonique et celles de la forme produite par les participants. Cette analyse concerne 3,6k mots produits par les participants. Les résultats montrent que les erreurs de prononciation augmentent de $10 \%$ dans la lecture oralisée par rapport à la tâche d'imitation. Nous trouvons que la compétence phonique ne change pas en fonction du niveau de langue. Nous faisons l'hypothèse que ces erreurs sont dues, outre le transfert phonétique-phonologie de la L1 sur la L2, aux interférences négatives de l'orthographe (associations erronées entre graphie et son). Nous discutons de l'impact négatif que peut avoir l'input écrit dans l'acquisition de la phonétique/phonologie en L2, facteur souvent ignoré dans les recherches en phonologie.

\section{ABSTRACT}

We report the effects of two types of tasks (imitation $v s$ reading), orthography and proficiency level on the pronunciation accuracy in the speech of 27 Mexican Spanish learners of L2 French. We calculate Levenshtein distances between the phonetic transcriptions of the canonical pronunciation in L1 French and the actual learners' productions as an evaluation of the pronunciation accuracy. The analysis was carried out on $3.6 \mathrm{k}$ words produced by the participants. Results show that pronunciation error rates increase by $10 \%$ in the reading task with respect to the imitation task. We did not find any effect of the proficiency level. We propose that these errors are due, apart from the L1 phonological/phonetic transfer, also to the negative L1 transfer of letter-to-sound correspondences on the L2. We discuss the negative effects of written input on the acquisition of L2 phonology (a factor that is neglected in current L2 phonology models).

MOTS-CLÉS : prononciation en FLE, erreurs de prononciation en FLE, orthographe du français.

KEYWORDS: pronunciation in L2 French, pronunciation errors in L2 French, French orthography.

\section{Introduction}

Les apprenants adultes d'une L2 en contexte formel (apprentissage de la langue cible dans un pays où la L2 n'est pas une langue officielle/parlée) sont souvent confrontés à l'input écrit depuis le début de l'acquisition. Il est donc fréquent que les apprenants/enseignants emploient, outre l'input auditif ou les exercices d'entraînement articulatoire, le support de l'écrit pour apprendre et enseigner la 
prononciation des nouveaux sons. En l'occurrence, les règles d'association entre les graphies et les sons dans la L2 sont souvent utilisées pour apprendre le nouveau système sonore de la L2.

L'apprenant adulte d'une L2 peut être influencé, non seulement par la phonétique et la phonologie de sa L1, mais également par les effets de l'orthographe de la L1 et de la L2 elle-même. Les apprenants sont donc confrontés à deux types de problèmes : la difficulté de prononcer et percevoir les nouveaux sons de la langue cible et la difficulté d'éviter une prononciation orthographique ou des associations erronées entre les graphies et les sons (cf. Detey et al. 2005, Young-Sholten 2002).

L'apprenant doit surmonter les inconsistances des rapports graphèmes-phonèmes en français L2, langue avec un système d'orthographe opaque (cf. Catach 2004) : (i) un phonème peut correspondre à différentes configuration graphiques (/o/ peut correspondre aux suites $<0>,<\mathrm{au}>$ ou $<$ eau $>$ ), (ii) une graphie peut correspondre à différents sons $(<\mathrm{s}>$ se prononce $[\mathrm{s}]$ dans le mot sel mais $[\mathrm{z}]$ dans le mot base), (iii) une graphie n'est pas prononcée $(<\mathrm{t}>$ dans le mot chat), etc. L'objectif de cette étude est d'examiner les effets de la lecture oralisée et de l'orthographe dans la prononciation du français L2 chez les apprenants hispanophones du français L2 au Mexique. Notre intérêt sera d'examiner l'impact que peuvent avoir les systèmes d'orthographe opaque, comme c'est le cas du français, dans l'acquisition de l'émergence de l'interlangue phonologique d'une L2.

\section{Le rôle de l'orthographe dans la prononciation en L2}

La place qu'occupe l'orthographe dans l'apprentissage de la prononciation a été largement négligée dans les nombreux modèles d'acquisition de la phonologie/phonétique des L2 (cf. le Speech Learning Model (Flege 1995), le Perceptual Assimilation Model (Best 1995) ou encore le Second Language Linguistic Perception Model (Escudero 2005), entre autres). Il faut noter que ce n'est pas le cas de l'influence de l'orthographe dans la prononciation en L1 chez les monolingues, car différentes études en psycholinguistique ont examiné l'influence de l'orthographe dans la reconnaissance des phonèmes/mots (Frauenfelder et al. 1990) et dans la variation phonétique (Chevrot \& Malderez 1999)

L'influence de l'orthographe dans la prononciation en L2 n'a attiré l'attention des chercheurs que depuis quelques années. Les études consacrées à ce sujet se classent en deux domaines. Le premier concerne l'interaction entre les représentations orthographiques de la L1/L2 dans le développement de l'interphonologie au niveau de la production. C'est le cas de l'influence des graphies dans la prononciation des approximantes en espagnol L2 par des anglophones : lorsque la suite en espagnol la vaca (la vache) est prononcée [la.'va.ka] au lieu de [la.'ßa.ka] dû à la présence de la graphie $<\mathrm{v}>$ (Zampini 1994). Un autre exemple est celui de l'influence des graphies silencieuses dans l'insertion des voyelles épenthétiques en anglais L2 par des lusophones du Brésil : lorsque le mot anglais tape est prononcé ['tej.pi] ou ['tej.pə] dû à la présence de la lettre finale <e> (Silveira 2007).

Le deuxième domaine s'intéresse à évaluer les effets de l'orthographe dans la perception/imitation en L2. Les travaux de Bassetti (2017) et Detey et al. (2005) vont dans ce sens. Ces séries de travaux ont mis en évidence que les effets de l'orthographe sont, en quelque sorte, durables dans la production orale, si bien que seulement les effets des graphies peuvent expliquer les erreurs en imitation ou reconnaissance des mots dans la langue cible. Ainsi, certaines erreurs de prononciation seraient motivées par des interférences de l'orthographe de la L1-L2, même lorsque les apprenants articulent la L2 en dehors de la présence de l'écrit. Tout ceci nous amène à considérer que, outre l'impact de la L1, l'orthographe peut avoir des effets sur l'acquisition de la phonologie/phonétique en L2. Bien que pour la plupart des théories en phonologie de L2, l'input auditif (perception), les compétences en production orale et le rôle de la L1 (entre autres) soient les facteurs les plus importants pour expliquer 
l'émergence de la structure sonore en L2, il est indéniable que les effets du visuel (et plus particulièrement les effets de l'orthographe sur la production orale) ont une incidence sur la prononciation de la L2.

\section{Questions de recherche et méthodologie}

Nous nous intéressons ici à étudier l'effet de la tâche (lecture $v s$ imitation) et de l'orthographe en français L2 dans la production orale des hispanophones. Nous nous posons 2 types de questions : (i) Les erreurs de prononciation se distribuent-elles différemment en fonction de la tâche réalisée (imitation $v s$ lecture) et/ou du niveau de langue des apprenants ? (ii) Quel est le rôle de l'orthographe dans l'émergence de ces erreurs?

\subsection{Protocole expérimental}

Les données ont été collectées à partir du protocole IPFC-espagnol (Racine et al. 2012) auprès de 27 étudiants universitaires hispanophones du Mexique. Les participants ont réalisé plusieurs tâches : une tâche d'imitation sans support écrit, deux tâches de lecture de mots avec un support écrit, la lecture d'un texte, un entretien semi-guidé et une production en binômes (avec un autre apprenant). Pour la présente étude, nous analysons la production orale des 68 mots conçus dans le protocole IPFCespagnol dans la tâche de lecture et d'imitation. Ces mots contenaient des phonèmes potentiellement difficiles pour les locuteurs hispanophones apprenant le français L2 (par exemple, $/ \mathrm{s} / \sim / \mathrm{z} /$ dans les mots hausse vs ose, $/ \mathrm{u} / \sim / \mathrm{y} /$ dans les mots boule vs bulle, l'articulation $\mathrm{du} / \mathrm{d} /$ en position initiale $v s$ intervocalique dans les mots dorer vs adorer, etc.). Dans la tâche d'imitation, les participants ont écouté une série de 68 stimuli (mots isolés) et avaient 5 secondes entre chaque mot pour le reproduire. La tâche de lecture consistait à lire les mêmes mots que dans la tâche d'imitation mais devant un écran. Pour cette tâche, les participants devaient lire l'input affiché sur l'écran d'un ordinateur avec 3 secondes d'intervalle entre chaque mot.

\subsection{Participants}

Les participants étaient formés de 27 étudiants mexicains (16 femmes et 11 hommes) qui poursuivant leurs cours de formation universitaire à l'Université Nationale Autonome du Mexique. Ils poursuivaient, parallèlement à leurs cours universitaires, des cours de français L2 dans 1'Ecole Nationale de Langues de cette université. Les étudiants ont été classés en deux groupes selon le niveau de leurs cours de français au moment de l'expérience : 9 étudiants intermédiaires ou A2 $(+)$ et 18 avancés ou B1(+). Leur âge moyen est de 23,9 ans (é.-t. 5,8). Tous les participants ont été enregistrés dans une pièce calme, dans les locaux de cette université.

\subsection{Annotation}

Le corpus analysé contient 3672 mots produits par les participants (68 mots x 2 tâches x 27 locuteurs). Deux phonéticiens ont encodé la production de tous les mots avec une transcription phonétique large (coarticulation exclue) de manière individuelle sous Praat. Ensuite, les annotateurs ont réalisé une correction manuelle avec la visualisation du spectrogramme. Enfin, dans une troisième étape, les annotations ont été confrontées afin de les harmoniser. En cas de divergences, les deux annotateurs se sont entendus sur une seule transcription. 


\subsection{Distances de Levenshtein}

L'évaluation de la prononciation a été obtenue à partir des scores obtenus avec les distances de Levenshtein, mesures utilisées dans d'autres études en acquisition de L2 (cf. Weiling et al. 2014). Ces distances ont été calculées à partir de la transcription phonétique de la production des apprenants et la production attendue (transcription phonétique standard du français parisien). Cet algorithme permet de calculer le degré de différence entre la transcription de la cible et la prononciation réelle. Le score obtenu rend compte du nombre d'insertions, suppressions ou remplacements nécessaires pour transformer la chaîne de caractères de la transcription de la prononciation en L2 en la chaîne de la prononciation cible. L'algorithme permet également d'aligner automatiquement les segments identiques entre les deux annotations.

Un exemple est illustré dans la FIGURE 1 avec les valeurs obtenues avec les mots hors et augmenter dans la tâche de lecture. Dans ces exemples, les lignes rouges symbolisent les remplacements de phonèmes et les points verts les ajouts. Pour chacun de ces changements (erreurs de prononciation de l'apprenant), l'algorithme fournit la valeur de 1, tandis que pour les alignements sans changements (prononciation canonique), la valeur est de 0.

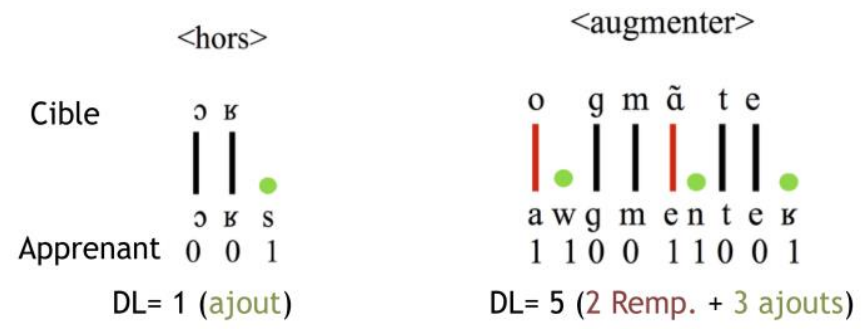

FIGURE 1: Exemple des valeurs obtenues via les distances de Levenshtein

Les distances ont été ensuite normalisées par rapport au nombre de phonèmes pour chaque mot afin d'éviter les effets de la variabilité du nombre de phones de chaque stimulus sur le nombre de changements nécessaires. Pour mesurer les effets de la tâche, nous avons calculé un pourcentage de similarité avec les distances de Levenshtein normalisées entre la production des apprenants et la prononciation normée.

Pour ce qui est des effets de l'orthographe, nous avons retenu l'analyse de la production de 4(archi)phonèmes en particulier, lesquels étaient associés à une graphie transparente/opaque : (i) / z/ écrit avec une graphie transparente $<$ zoo $>v s$ opaque $<$ base $>$, (ii) $/ \mathrm{O} /$ écrit avec une graphie transparente $<$ port $>v s$ opaque $<$ peau $>$, (iii) $/ \mathrm{u} /$ écrit avec une graphie opaque $<$ boule $>$ et (iv) $/ \mathrm{y} /$ écrit également avec une graphie opaque $<$ bulle $>$. Il faut noter que l'opacité de ces deux derniers phonèmes est déterminée en fonction de la L1 des apprenants. En effet, les graphies $<$ ou $>$ et $<u<$ correspondent aux sons [ou] et [u] respectivement en espagnol, mais aux sons [u] et [y] en français L2.

\section{Résultats}

Les données ont été analysées moyennant des modèles linéaires mixtes (Baayen 2008) avec le package lme4 sous R (Bates et al. 2015). Les constantes (intercepts) aléatoires pour les locuteurs et les mots ont été évaluées dans ces modèles, ainsi que les pentes aléatoires par locuteur et par mot. La 
puissance des variables prédictives a été estimée moyennant des tests de rapport de vraisemblance entre les modèles avec et sans les effets fixes.

\subsection{Effets de la lecture et du niveau}

La première question concernait la distribution des erreurs en fonction de la tâche et du niveau des apprenants. La FIGURE 2 montre que la lecture paraît affecter de manière négative la prononciation chez les participants. En revanche, ce n'est pas le cas pour les effets du niveau où nous pouvons observer que les pourcentages de similarité ne diffèrent pas entre les étudiants A2 et B1.

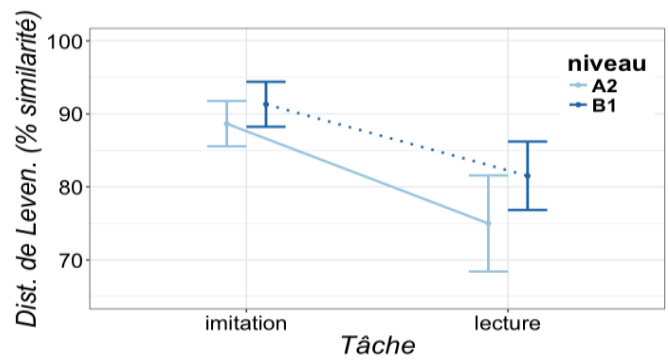

FIGURE 2: Effets de la tâche et du niveau dans la prononciation en français L2

Afin de confirmer ces observations, nous avons construit un modèle à effets mixtes (multinomial). Dans ce modèle, nous avons entré les valeurs des POURCENTAGES DE SIMILARITÉ selon les distances de Levenshtein comme variable dépendante, le NIVEAU des apprenants (A2 vs B1), la TACHE (imitation $v s$ lecture) comme effets fixes, et les participants et les mots comme effets aléatoires. Les résultats montrent que le pourcentage moyen de similarité entre la prononciation réelle des étudiants et la prononciation normée est de $90,5 \%$ dans la tâche d'imitation, alors qu'en lecture, le degré de similarité descend à 79,9\%. Les résultats statistiques ont confirmé que la TÂCHE a un effet sur la prononciation des apprenants $\left(\chi^{2}(2)=28,17, p<0,0001\right)$. Autrement dit, la lecture fait augmenter les erreurs de prononciation de $10 \%$ selon nos métriques. Pour ce qui est du NIVEAU, les résultats montrent que les étudiants $\mathrm{B} 1$ arrivent à reproduire les mots avec la prononciation attendue avec $86,2 \%$ de similarité, tandis que les étudiants A2 le font avec un taux de similarité de $82,2 \%$. Les résultats du modèle statistique montrent, en revanche, que cette différence n'est pas statistiquement significative $(p>0,05)$. Les résultats du modèle évaluant les effets d'une interaction TACHE*NIVEAU sur les POURCENTAGES DE SIMILARITÉ n'ont pas atteint le seuil de significativité non plus $(p>0,05)$. En d'autres mots, les effets négatifs de la TÂCHE (lecture) dans la prononciation sont similaires pour les deux niveaux de langue testés (cf. la similitude des pentes de la FIGURE 2).

Ces observations démontrent que les étudiants ne semblent pas améliorer leur prononciation selon le niveau. De fait, ces résultats montrent que les étudiants B1 sont autant affectés que les étudiants A2 par la tâche de lecture. Ces résultats confirment ce qui avait été soulevé par les études précédentes : les participants montrent une certaine sensibilité à la présence des graphies lors des tâches de lecture oralisée. En plus, cette influence de l'écrit sur l'oral ne semble pas diminuer avec l'apprentissage de la L2. 

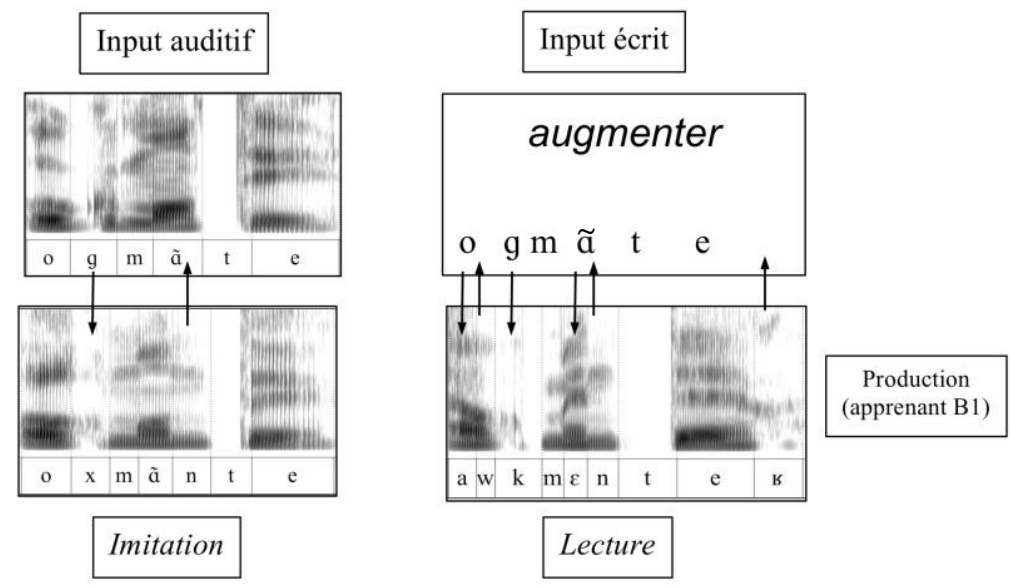

FIGURE 3: Ajouts et remplacements des phones pour le mot augmenter selon la tâche

La FIGURE 3 illustre les effets de la tâche dans nos données. Nous pouvons constater que le nombre de remplacements (flèches vers le bas) et ajouts (flèches vers le haut) s'élèvent dans la tâche de lecture par rapport à la tâche d'imitation. Cette analyse montre que la présence de l'input écrit modifie négativement la prononciation chez les participants. Dans cet exemple, il est intéressant de noter que le nombre d'insertions de phones déclenchés par la lecture est très probablement dû à une association erronée de la production oralisée des graphies silencieuses dans l'input écrit. Cette analyse réside dans le fait que l'insertion et le remplacement des phones de cet exemple ne peuvent pas être directement attribuables à l'influence de la phonétique/phonologie de la L1. A tout le moins, le remplacement de la voyelle [o] par la suite [aw], et l'ajout du [ $\mathrm{b}]$ final dans le mot $<$ augmenter $>$ dans la tâche de lecture peut être difficilement attribuable à l'influence de la phonologie/phonétique de la L1 de l'apprenant, mais plutôt à une mauvaise association des graphies avec les sons.

\subsection{Effets de l'orthographe}

Le deuxième objectif de cette étude était d'examiner les effets de l'orthographe à partir de la réalisation des phonèmes associés à des graphies transparentes $v s$ opaques. Notre hypothèse était que la présence d'une graphie opaque devrait déclencher des erreurs de prononciation, à la différence de la présence d'une graphie transparente, laquelle devrait favoriser la prononciation. attendue. Si c'était le cas, nous pourrions donner des arguments pour montrer que les erreurs des apprenants (remplacement d'un phonème par un autre) sont induites exclusivement par la présence de la graphie. Ainsi, nous avons évalué si la prononciation du phonème /z/ émergeait comme $[\mathrm{z}]$ dans les mots où un tel phonème était associé à une graphie transparente $(<\mathbf{z o o}>)$ vs opaque $(<$ base $>$ ) dans la tâche de lecture $(5$ mots pour chaque cas). La même hypothèse était formulée pour l'articulation de l'archiphonème $/ O /:$ nous avons comparé les mots où cet archiphonème était associé à une graphie transparente $(/ \mathrm{J} /$ pour $<$ port $>)$ vs opaque (/o/ pour < peau $>$ ) par rapport à la L1 de l'apprenant (4 mots différents pour chaque cas).

Les phonèmes français $/ \mathrm{u} / v_{s} / \mathrm{y} /$ seraient tous les deux associés à des graphies opaques pour l'apprenant selon les règles de leur L1 ( $v$. supra). Nous avons donc comparé si les effets de l'orthographe étaient les mêmes pour chacun de ces cas $(<$ boule $>$ vs $<$ bull $>, 3$ mots différents pour chaque phonème. 
La FIGURE 4 montre les pourcentages moyens de réussite entre le phonème produit par les participants et la cible attendue en fonction des graphies transparentes (bleu clair) et opaques (bleu foncé). Cette figure montre que les graphies opaques ont des effets négatifs dans la prononciation comme il était attendu, car les taux de succès descendent dans les deux cas.
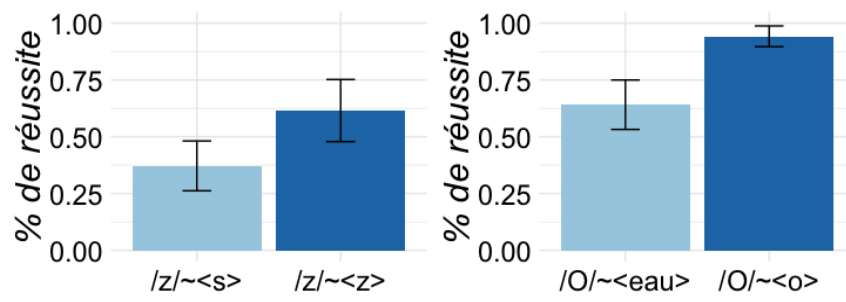

FIGURE 4: Effets des graphies opaques vs transparentes dans la prononciation en français L2

Nous avons construit différents modèles à effets mixtes (binomiaux) afin de corroborer ces observations où nous avons entré POURCENTAGES DE RÉUSSITE (phonème correct vs incorrect) comme variable dépendante, NIVEAU (A2 vs B1) et GRAPHIES (transparente $v s$ opaque) comme variables fixes, et participants et mots comme effets aléatoires. Les résultats statistiques montrent un effet des GRAPHIES : les erreurs de prononciation augmentent avec la lecture des graphies opaques par rapport aux graphies transparentes $\left(\chi^{2}(2)=8,87, p<0,01\right)$. Ainsi, le phonème /z/ est articulé comme [z] dans $61 \%$ des cas avec la graphie transparente $<\mathrm{Z}>$ contre $37 \%$ avec la graphie opaque $<$ s $>$, laquelle a été prononcée massivement comme [s]. Les effets du NIVEAU ne sont pas statistiquement significatifs $(p$ $>0,05)$, cela montrant que les deux groupes ont des taux de réussite similaires $(49 \%$ pour A2, et $50 \%$ pour B1). Les résultats montrent que les effets de l'interaction NIVEAU*GRAPHIE n'atteignent pas non plus le seuil de significativité $(p>0,05)$. En d'autres termes, les effets des GRAPHIES sur la prononciation sont similaires dans les deux groupes d'apprenants.

Pour le cas de l'archiphonème /O/, il est articulé, soit comme [o] ou soit comme [0], dans $94 \%$ des cas lorsque ce phonème est associé à la graphie $<_{0}>$ contre $64 \%$ des cas avec les graphies $<$ eau $>$, contexte où les apprenants ont produit le son [ø] dans la plupart des cas. Le test statistique évaluant les effets du facteur GRAPHIES montre que les graphies opaques nuisent à la prononciation de cet archiphonème $\left(\chi^{2}(2)=35,83, p<0,0001\right)$. Pour ce qui est des effets du NIVEAU, le taux de réussite est de $73 \%$ pour le groupe A2 et de $84 \%$ pour le groupe B1. Toutefois, ces différences ne sont pas statistiquement significatives $(p>0.05)$. Les résultats du modèle statistique évaluant l'interaction NIVEAU*GRAPHIES n'atteignent pas le seuil de significativité $(p>0,05)$, cela montrant que les effets négatifs des graphies opaques affectent de manière similaire la prononciation de cet archiphonème dans les deux groupes d'apprenants.

Enfin, nous avons analysé dans quelle mesure les deux types de graphies opaques selon la L1 des apprenants avaient une incidence dans la prononciation et avons examiné si l'une d'entre elles s'avéraient comme plus problématique pour les apprenants. C'est le cas de la production du phonème $/ \mathrm{u} /$ et $/ \mathrm{y} /$ associés aux graphèmes $<\mathrm{ou}>$ et $<\mathrm{u}>$ respectivement. Les résultats montrent un effet des GRAPHIES : la graphie $<\mathrm{u}>$ fait augmenter les erreurs de prononciation par rapport aux graphies $<$ ou $>$ $\left(\chi^{2}(2)=8,55, p<0,01\right)$. La production du phonème $/ \mathrm{u} /$ émerge comme $[\mathrm{u}]$ dans $88 \%$ des cas alors que le phonème $/ \mathrm{y} /$ émerge comme $[\mathrm{y}]$ dans $51 \%$ des cas. Ceci montre que le graphème $<$ ou $>$ trouble moins les participants que le graphème $<\mathrm{u}>$ où ce dernier était massivement articulé comme $[\mathrm{u}]$. Ceci contraste avec les résultats de la tâche d'imitation, où le phonème /y/ émerge comme [y] dans le $96 \%$ 
des cas. Les résultats montrent aussi que le facteur NIVEAU n'a pas d'effets dans la prononciation ( $p$ $>0,05$ ). Les valeurs de $p>0,05$ du test statique évaluant les effets de l'interaction NIVEAU*GRAPHIES montre que, comme dans les deux cas précédents, les effets négatifs de ces deux graphies sur la prononciation sont similaires dans les deux groupes d'apprenants.

\section{Discussion \& Conclusion}

Les résultats reportés dans les sections précédentes permettent de faire plusieurs constatations. D'une part, la tâche de lecture a des effets négatifs sur la prononciation des apprenants : elle peut déclencher une mauvaise association des graphies-sons, ou bien l'insertion de phonèmes due à la présence de graphies silencieuses. D'autre part, nos résultats montrent que le niveau de compétence phonique dans les deux groupes est relativement homogène. Cela est d'autant plus surprenant que la différence de niveau de langue est relativement importante : $\mathrm{A} 2(+)$ vs $\mathrm{B} 1(+)$. Ces observations suggèrent que les compétences de prononciation des apprenants hispanophones selon leur niveau de langue semblent être immuables. De fait, nos résultats montrent que l'influence négative de la tâche de lecture ne diminue pas avec l'apprentissage de la L2.

Ensuite, nous avons trouvé que les graphies opaques déclenchent des erreurs de prononciation pour les quatre phonèmes étudiés ici. Le fait que le remplacement des phones augmente avec les graphies opaques en rapport avec les graphies transparentes confirme qu'il existe des rapports entre la compétence phonétique et l'orthographe. Nous montrons que, dans tous les cas, le niveau des apprenants n'a aucune incidence dans la prononciation : les effets négatifs de l'orthographe affectent de manière similaire les deux niveaux testés. Ainsi, il semblerait que les apprenants B1 ne semblent pas surmonter les interférences de l'orthographe par rapport aux étudiants A2.

Finalement, si les résultats présentés ici n'abordent pas tous les phonèmes en français, ni toutes leurs possibilités de représentation orthographique, ils confirment que la compétence phonique n'est pas dissociée des compétences visuelles, en l'occurrence, l'association graphies-sons. Ces résultats permettent d'avancer la question suivante : le transfert phonologique négatif de la L1 est-il renforcé par la présence de l'écrit? L'un des objectifs de notre future recherche est de mieux examiner les traits phonologiques concernés dans le transfert de la L1-L2 attribuables à la phonologie et à l'orthographe. Cela permettrait d'avoir des explications plus claires sur les effets de l'orthographe dans le développement de la compétence phonétique en français L2. Il n'en reste pas moins que nous souhaitons souligner les effets positifs de la tâche d'imitation : la perception auditive semble être en définitive une technique qui n'entraînerait que des bénéfices dans l'apprentissage de la structure sonore de la L2.

\section{Références}

BAAYEN, R.H. (2008). Analyzing linguistic data: A practical introduction to statistics using $R$. Cambridge : Cambridge University Press.

BASSETTI, B. (2017). Orthography affects second language research: double letters and geminate production in English. Journal of Experimental Psychology: Learning, Memory and Cognition, 43(11), 1835-1842.

Bates, D., MAechler, M., Bolker, B. \& Walker, S. (2015). Fitting Linear Mixed-Effects Models using lme4. Journal of Statistical Software, 67(1), 1-48. 
BEST, C.T. (1995). A direct realist view of cross-language speech perception. In W. STRANGE (éd.), Speech perception and linguistic experience: Theoretical and methodological issues in crosslanguage speech research. Timonium, MD: York Press, 171-204.

CATACH, N. (1991). L'orthographe. Paris: PUF, 9e édition.

ChEVRot, J.-P. \& MALDEREZ, I. (1999). L'effet Buben : de la linguistique diachronique à l'approche cognitive (et retour). Langue française, 124, 104-125.

Detey, J., DuRAnd J. \& Nespoulous J.-L. (2005). Interphonologie et représentations orthographiques. Le cas des catégories $/ \mathrm{b} /$ et $/ \mathrm{v} /$ chez des apprenants japonais de Français Langue Etrangère. Revue Parole, 34-36, 140-185.

ESCUdERO, P. (2005). Linguistic Perception and Second Language Acquisition: Explaining the attainment of Optimal Phonological Categorization. Thèese de Doctorat. Université d'Utrecht.

FLEGE, J.E. (1995). Second language speech learning: Theory, findings and problems. In W. STRANGE (éd.), Speech perception and linguistic experience: Theoretical and methodological issues in crosslanguage speech research. Timonium, MD: York Press, 233-277.

FRAUENFELder, U.H., Segui, L. \& DiJKSTRA, T. (1990). Lexical effects in phonemic processing: facilitatory or inhibitory? Journal of Experimental Psychology: Human Perception and Performance, $16(1), 77-91$.

RACiNe, I., DETEY, S., ZAY, F. \& KAWAGUCHI, Y. (2012). Des atouts d'un corpus multitâches pour l'étude de la phonologie en L2: l'exemple du projet "Interphonologie du français Contemporain" (IPFC). In KAMBER, A., SKUPIENS, C. (éd.), Recherches récentes en FLE. Berne: Peter Lang, 1-19.

SILVEIRA, R. (2007). The role of task-type and orthography in the production of word-final consonants. Revista de Estudos da Linguagem, 15(1), 143-176.

Young-SchOlten, M. (2002). Orthographic input in L2 phonological development. In. P. BURMEISTER \& A. RHODE (éd.), An integrated view of language development: Papers in honour of Henning Wode. Trier, Allemagne: Verlag, 263-279.

WIEILING, M. et al. (2014). Measuring foreign accent strength in English : Validating Levenshtein Distance as a Measure. Language Dynamics and Change, 4(2), 253-269.

ZAMPINI, M. (1994). The role of native language and tasks formality in the acquisition of Spanish Spirantization. Hispania, 77(3), 470-481. 